\title{
ELABELA attenuates deoxycorticosterone acetate/ salt-induced hypertension and renal injury by inhibition of NADPH oxidase/ROS/NLRP3 inflammasome pathway
}

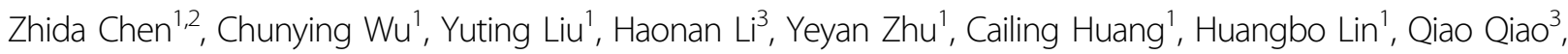
Mengming Huang ${ }^{3}$, Qing Zhu ${ }^{3}$ and Lei Wang ${ }^{1}$

\begin{abstract}
ELABELA (ELA), a 32-residue hormone peptide abundantly expressed in adult kidneys, has been identified as a novel endogenous ligand for APJ/Apelin receptor. The aim of this study was to investigate the role of ELA in deoxycorticosterone acetate (DOCA)/salt-induced hypertension and further explore the underlying mechanism. In DOCA/salt-treated rats, the mRNA level of ELA greatly decreased in the renal medulla. Next, overexpression of ELA in the kidney was found to attenuate DOCA/salt-induced hypertension and renal injury, including lower blood pressure, reversed glomerular morphological damage, decreased blood urea nitrogen (BUN), and blocked the accumulation of fibrotic markers. Mechanistically, ELA overexpression inhibited renal nicotinamide adenine dinucleotide phosphate (NADPH) oxidase activity and subsequent reactive oxygen species (ROS) production, thus resulted in the blockade of formation and activation of Nod-like receptor protein 3 (NLRP3) inflammasome. The inhibitory effects of ELA on Aldosterone-stimulated NADPH oxidase/ROS/NLRP3 inflammasome pathway were confirmed in the human renal tubular cells. Furthermore, our in vivo and in vitro results showed that the deficiency of the apelin receptor APJ did not influence the antihypertensive effect and blockage to NADPH oxidase/ROS/ NLRP3 pathway of ELA. Moreover, in heterozygous ELA knockout mice $\left(E L A^{+/-}\right.$), the ELA deficiency remarkably accelerated the onset of DOCA/salt-induced hypertension. Our data demonstrate that ELA prevents DOCA/saltinduced hypertension by inhibiting NADPH oxidase/ROS/NLRP3 pathway in the kidney, which is APJ independent. Pharmacological targeting of ELA may serve as a novel therapeutic strategy for the treatment of hypertensive kidney disease.
\end{abstract}

\section{Introduction}

Both human and animal studies demonstrate that high level of dietary salt contributes to the development of hypertension ${ }^{1}$. It is found that excessive salt intake leads to

Correspondence: Qing Zhu (zyq973@hotmail.com) or

Lei Wang (wanglei_voice@yahoo.com)

${ }^{1}$ School of Pharmaceutical Sciences, Guangzhou University of Chinese

Medicine, Guangzhou, China

${ }^{2}$ Department of Nephrology, The Second Affiliated Hospital, School of

Medicine, Zhejiang University, Hangzhou, China

Full list of author information is available at the end of the article

Edited by F. Pentimall the imbalance of volume homeostasis and sodium reabsorption, which ultimately results in the elevation of blood pressure $(\mathrm{BP})^{2}$. During this process, kidney is a major organ for BP homeostasis. Recently, there is a growing body of evidence suggesting that inflammation plays a crucial role in the progression of salt-sensitive hypertension as well as kidney dysfunction ${ }^{3-5}$. In experimental models, salt-sensitive hypertension are associated with the accumulation and activation of $\mathrm{T}$ cells in the kidney, which produces inflammatory cytokines including IFN- $\gamma$, IL-17, and TNF- $\alpha$; results in sodium retention, vasoconstriction

\section{(c) The Author(s) 2020}

(c) (i) Open Access This article is licensed under a Creative Commons Attribution 4.0 International License, which permits use, sharing, adaptation, distribution and reproduction cc) in any medium or format, as long as you give appropriate credit to the original author(s) and the source, provide a link to the Creative Commons license, and indicate if changes were made. The images or other third party material in this article are included in the article's Creative Commons license, unless indicated otherwise in a credit line to the material. If material is not included in the article's Creative Commons license and your intended use is not permitted by statutory regulation or exceeds the permitted use, you will need to obtain permission directly from the copyright holder. To view a copy of this license, visit http://creativecommons.org/licenses/by/4.0/. 
and enhanced oxidative stress; and then promotes hypertension, glomerular injury, renal fibrosis, and dysfunction ${ }^{6}$.

Inflammasomes are known as upstream machineries to trigger inflammation in response to danger signals from pathogens or damaged host ${ }^{7,8}$. Among them, Nod-like receptor protein 3 (NLRP3) inflammasome is best characterized, which consists of three major proteins: NLRP3, the adaptor protein ASC, and the effector caspase-1. Once activated, the inflammasome recruits these three components and cleaves pro-caspase- 1 to an active form (cleavage caspase-1) which triggers proteolytic cleavage of pro-IL-1 $\beta$ and pro-IL-18 to mature and secreted forms ${ }^{9,10}$. NLRP3 inflammasome has been reported to play a fundamental role in the pathogenesis of saltsensitive hypertension ${ }^{4,7,11,12}$. The mice with NLRP3 inflammasome deficiency failed to increase BP upon the DOCA/salt treatment ${ }^{7,11}$. It is known that there are several pathways to activate the NLRP3 inflammasome in response to diverse endogenous and exogenous danger signals, such as reactive oxygen species $(\mathrm{ROS})^{13-15}$. Nicotinamide adenine dinucleotide phosphate (NADPH) oxidase is a key enzyme to generate superoxide intracellularly. The activation of NADPH oxidase is initiated by the assembly of cytosolic subunits, $\mathrm{p} 22^{\text {phox }}$, and gp91 ${ }^{\text {phox }}$ within the membrane while $\mathrm{p} 47^{\text {phox }}$ and $\mathrm{p} 67^{\text {phox }}$ in the cytoplasm. $\mathrm{p} 22^{\text {phox }}$ and gp91 $1^{\text {phox }}$ are found to help electron transfer from NADPH to oxygen molecules yielding $\mathrm{O}_{2}{ }^{-16} \cdot \mathrm{p} 47^{\text {phox }}$ is able to interact with the cytoplasmic tail of $\mathrm{p} 22^{\text {phox }}$ and $\mathrm{p} 67^{\text {phox }}$ binds to gp91 ${ }^{\text {phox }}$, resulting in the recruitment of related enzyme complex $^{17,18}$. Previous studies show that NADPH oxidase inhibitor apocynin or deficiency of gp $91^{\text {phox }}$ or $\mathrm{p} 47^{\text {phox }}$ attenuates the hypertensive response in the DOCA/salttreated mice ${ }^{19,20}$, demonstrating that NADPH oxidase activity contributes to DOCA/salt-induced hypertension. In addition, inhibition of the NADPH oxidase subunit gp91 ${ }^{\text {phox }}$ or $\mathrm{O}_{2}{ }^{-}$scavenging suppresses NLRP3 inflammasome activation and ultimately ameliorates renal injury ${ }^{14,21}$. Taken together, these findings suggest that the $\mathrm{NADPH}$ oxidase/ROS/NLRP3 pathway serves as a key pathogenic mechanism of salt-sensitive hypertension.

ELABELA/Toddler/Apela (ELA) is a 32-amino-acid peptide as a novel endogenous ligand for the APJ/Apelin receptor ${ }^{22}$. Previous studies demonstrate that ELA plays diverse biological functions in embryonic development ${ }^{22-25}$. Actually, the expression of ELA is also detected abundantly in adult kidneys ${ }^{26,27}$, especially in renal medulla $^{28}$, suggesting that ELA may have important roles in the kidney. Recently, ELA and its shortest furin-cleaved fragment ELA11 are found to have protective effects on hypoxia-reperfusion $(H / R)$ induced renal inflammation and fibrosis ${ }^{27}$. Another group reveals that ELA regulates water homeostasis by binding to the APJ receptor ${ }^{26}$. Furthermore, exogenous ELA treated in heart is found to exhibit antihypertensive effects in angiotensin II-infused mice, high salt fed Dahl salt-sensitive rats and rodent pulmonary arterial hypertension models ${ }^{28-30}$. However, whether ELA in kidney may exhibit antihypertensive and reno-protective actions in salt-sensitive hypertension remains unknown. The present study was aimed to investigate the roles of ELA in the regulation of BP and renal injury in DOCA/salt-treated animals as well as the potential molecular mechanism involved.

\section{Methods \\ Animals}

Male Sprague-Dawley (SD) rats, C57BL/6 mice, and heterozygous ELA knockout C57BL/6 mice $\left(\mathrm{ELA}^{+-}\right)$ were used in this study. Male SD rats and C57BL/6 mice were purchased from the animal center of Guangdong Province (Guangzhou, China). The ELA ${ }^{+/-}$heterozygous mice were generated by the Viewsolid Biotech Company (Beijing, China). The bodyweight of rats and mice were $250-300 \mathrm{~g}$ and $20-25 \mathrm{~g}$ respectively. All animals were cage housed with free access to tap water and standard chow, and maintained in a temperature-controlled room with 12: $12 \mathrm{~h}$ light-dark cycle. All the animals were randomly divided into groups as needed and euthanized with an excess intravenous dose of pentobarbital sodium (150 mg/kg, Sigma, 57-33-0) after experiments. The animal protocols were approved by the Institutional Animal Care and Use Committee of Guangzhou University of Chinese Medicine.

\section{Induction of hypertension and monitoring of blood pressure}

Hypertension was induced in rats or mice by the subcutaneous implantation of DOCA pellets $(150 \mathrm{mg} / \mathrm{rat}$ or $50 \mathrm{mg} /$ mouse) 1 week after unilateral nephrectomy. One percent $\mathrm{NaCl}$ drinking water was provided to all the DOCA treated animals, while control animals were maintained on tap water. Rat BP was monitored via the telemetry system (Data Science International, St Paul, Minnesota, USA). Mouse BP was measured by the tail cuff method (Kent Scientific Corporation, Torrington, CT, USA).

\section{Intrarenal ELA overexpression}

The ELA overexpression plasmid was constructed based on the commercial vector pcDNA3.1 (Invitrogen, V79020) which carries a constitutive promoter. Briefly, the histag fused ELA fragment of rat was amplified with the primers CGCGGATCCATGAGATTCCAGCCCCTTTT TTG and CGGAATTCTCAATGATGATGATGATGAT GGTCGACGGATGGGAAGGGCACTCGAGAAT and cloned into the BamHI-EcoRI site of pcDNA3.1, yielding the ELA overexpression plasmid pWRELAe-his. The constructed plasmid was confirmed by DNA sequencing (TsingKe Biological Technology, Beijing, China). 
The intrarenal transfection of ELA in rats was performed with pWRELAe-his in combined with the transfection reagent in vivo-jetPEI (Polyplus Transfection, PT101-01N), a polyethylenimine derivative, as previously described $^{31}$. Briefly, the left kidney was exposed from the flank region and an interstitial infusion catheter was placed into the renal medulla, $\sim 4-5 \mathrm{~mm}$ underneath the kidney surface, and secured using $3 \mathrm{~mol} / \mathrm{l}$ Vet bond tissue adhesive (3M, 1469SB) and a small piece of fat tissue. Then, $50 \mu \mathrm{g}$ of vehicle or pWRELAe-his were mixed with $8 \mu \mathrm{l}$ of in vivo-jetPEI in $5 \%$ glucose $(600 \mu \mathrm{l})$ and infused into the renal medulla $(20 \mu \mathrm{l} / \mathrm{min})$ through the infusion catheter. After infusion, the catheter was cut and blocked with fat tissue and Vetbond Tissue Adhesive.

\section{Assay of serum BUN, and collection of kidneys}

At the endpoint of experiment, blood samples were collected from abdominal aorta while rats were anesthetized with $2 \%$ isoflurane. The serum BUN (MLBio, ml623080) and IL-1 $\beta$ (MLBio, ml003057) were tested by the commercial available ELISA kits. Kidneys were removed and cut longitudinally. Half of the kidney was fixed in $4 \%$ paraformaldehyde and the other half dissected into cortex and medulla, frozen in liquid $\mathrm{N}_{2}$, and stored at $-80^{\circ} \mathrm{C}$.

\section{Cell culture and treatments}

The human renal tubular duct epithelial cells HK2 were purchased from the Procell Life Science \& Technology Company, Wuhan, China (CL-0109). The HK2 cells were grown in Dulbecco's modified Eagle Medium F12 (Gibco, C11330500BT) supplemented with 10\% (V/V) fetal bovine serum (HyClone, SV30160.03) and 1\% antibiotics (Procell, PB180120) in tissue culture flasks. The cells were pretreated for $1 \mathrm{~h}$ with ELA peptide $(1 \mathrm{nM}$, IGE Biotech, Guangzhou, China) followed by Aldosterone $(0.1 \mu \mathrm{M}$, Sigma, A9477) treatment. After $24 \mathrm{~h}$, cells were harvested for the analysis of gene expression, ROS production, and $\mathrm{NADP}^{+} / \mathrm{NADPH}$.

\section{Morphological analysis}

For morphological analysis, paraffin-embedded kidney sections $(4 \mathrm{~mm})$ were stained by hematoxylin-eosin. Glomerular damage was morphologically evaluated by two independent examiners who were blinded to the animal groups and semiquantitatively scored based on the degree of glomerular damage as described previously ${ }^{32}$. The average scores from counted glomeruli were used as the glomerular damage index for each animal.

\section{Quantitative reverse transcriptase PCR}

For quantitative reverse transcription PCR (qRT-PCR), total RNA isolation was performed as previously described $^{33}$. Reverse transcription and SYBR green based
Table 1 Primers used to quantify genes expression in this work.

\begin{tabular}{|c|c|c|}
\hline Gene & Species & Primer sequences \\
\hline \multirow[t]{2}{*}{ ELA } & Mouse & Sense: CAGAAACCAGTTAACTITCCCAGG \\
\hline & & Antisense: TGGGAAGGGCACTCGAGAAT \\
\hline \multirow[t]{2}{*}{ ELA } & Rat & Sense: GCGATGAGTCTCCTITITATCACG \\
\hline & & Antisense: TGGGAAGGGCACTCGAGAAT \\
\hline \multirow[t]{2}{*}{ NLRP3 } & Rat & Sense: CAGAAGCTGGGGTTGGTGAA \\
\hline & & Antisense: CCCATGTCTCCAAGGGCATT \\
\hline \multirow[t]{2}{*}{$\mathrm{IL}-1 \beta$} & Rat & Sense: TCGGCCAAGACAGGTCGCTCA \\
\hline & & Antisense: TGGTTGCCCATCAGAGGCAAGG \\
\hline \multirow[t]{2}{*}{ IL18 } & Rat & Sense: GGACTGGCTGTGACCCTATC \\
\hline & & Antisense: TGTGTCCTGGCACACGTTIT \\
\hline \multirow[t]{2}{*}{ MCP-1 } & Rat & Sense: CAGCCAGATGCAGTTAATGCC \\
\hline & & Antisense: AGCCGACTCATTGGGATCAT \\
\hline \multirow[t]{2}{*}{ P22phox } & Rat & Sense: GCCATTGCCAGTGTGATCTA \\
\hline & & Antisense: CTCCTCTTCGGCCTCACTT \\
\hline \multirow[t]{2}{*}{ P47phox } & Rat & Sense: CAGAATGTTGCCTGGTTG \\
\hline & & Antisense: GTCCCCTCCCTTAGATGA \\
\hline \multirow[t]{2}{*}{ P67phox } & Rat & Sense: TCTAAGAAGCTGGCGCTCTC \\
\hline & & Antisense: GCGTCTGAGTTTTCCCTTTG \\
\hline \multirow[t]{2}{*}{ gp91phox } & Rat & Sense: CTTCACACGGCCATTCACAC \\
\hline & & Antisense: GTCATAGGAGGGTTTCCGGC \\
\hline \multirow[t]{2}{*}{ GAPDH } & Rat & Sense: GTCTTCACTACCATGGAGAAGG \\
\hline & & Antisense: TCATGGATGACCTTGGCCAG \\
\hline \multirow[t]{2}{*}{ APJ } & Human & Sense: TCAGCAGCTACCTCATCTTC \\
\hline & & Antisense: ACTGCACCTTAGTGGTGTC \\
\hline \multirow[t]{2}{*}{ GAPDH } & Human & Sense: GATGACATCAAGAAGGTGGTG \\
\hline & & Antisense: GCTGTAGCCAAATTCGTTGTC \\
\hline
\end{tabular}

quantitative polymerase chain reaction were performed as the manufacturer's instructions (TsingKe Biological Technology, TSE202). Primers used in this work are shown in Table 1.

\section{Western blotting}

Kidney tissues or cells were lysed and subsequently sonicated in PBS that contained 1\% Triton X-100, $250 \mathrm{mmol} / \mathrm{L}$ phenylmethanesulfonyl fluoride, $2 \mathrm{mmol} / \mathrm{L}$ EDTA, and $5 \mathrm{mmol} / \mathrm{L}$ dithiothrietol (pH7.5). The total protein concentration was then determined by the BCA protein assay reagent kit (GBC, G3422). Thirty milligrams of protein for each sample was denatured in boiling water for $5 \mathrm{~min}$ then separated by SDS-PAGE and transferred onto polyvinylidene difluoride (PVDF) membranes. The 
blots were blocked $1 \mathrm{~h}$ with $5 \%$ nonfat dry milk in Trisbuffered saline, followed by incubation overnight with rabbit $\alpha$-smooth muscle actin ( $\alpha$-SMA) antibody (Affinity, AF1032), Collagen IA (Affinity, AF7001), NLRP3 (Abcam, ab214185), Caspase-1 (Affinity, AF4005), APJ (Fitzgerald, 70R-51439), p47phox (Affinity, AF5220) or p22phox (Affinity, DF10099) at $4{ }^{\circ} \mathrm{C}$. For $\beta$-actin, the membranes were stripped and reprobed with rabbit anti- $\beta$-actin antibody (Affinity, AF7018) or anti-GAPDH antibody (Affinity, AF7021). After being washed with Tris-buffered saline, membranes were incubated with a secondary antibody labeled with horseradish peroxidase-conjugated secondary antibody (Affinity, S0001) and visualized using enhanced chemiluminescence. The intensities of blotted bands were quantified with the software (ImageJ, free download from http://rsbweb.nih.gov/ij/).

\section{Immunohistochemical analysis}

For immunostaining, the slides were incubated in $0.3 \%$ $\mathrm{H}_{2} \mathrm{O}_{2}$ diluted in $100 \% \mathrm{MeOH}$ for 30 min to block endogenous peroxidase activity. The sections were incubated at room temperature for $30 \mathrm{~min}$ in PBS containing 1\% BSA (Sigma, 9048-46-8) to block nonspecific binding and then incubated overnight at $4{ }^{\circ} \mathrm{C}$ in a humidified chamber with an antibody against His-tag (Affinity, T0009) or $\alpha$ SMA diluted 1:500 in PBS containing 1\% BSA. Then the slides were incubated for $30 \mathrm{~min}$ at room temperature in a humidified chamber with anti-mouse IgG antibody (Vector Laboratories, BA-9200) and then HRP conjugated streptavidin (Boster, BA1088) in PBS. Then the slides were incubated with $50 \mu \mathrm{l}$ of diaminobenzadine (Vector Laboratories, SK4100) as a substrate, counterstained with hematoxylin (LEAGene, DH0006), dehydrated, and fixed with permount histological neutral balsam.

\section{Picro-sirius red staining for renal total collagen analysis}

Picro-sirius red staining was used to detect collagen accumulation. The paraffin-embedded heart or kidney samples were deparaffinised with alcohol and xylene. After three washes with distilled water, renal tissues were stained in Sirius red solution for $1 \mathrm{~h}$, washed in acetic acid, quickly dehydrated, and then mounted in a resinous medium. The extent of reactivity for Sirius red was quantified using image analysis software (Image-Pro Plus).

\section{Confocal microscopy of inflammasome proteins in HK2 cells}

The cells adherent to round glass coverslips were fixed with $4 \%$ buffered paraformaldehyde and permeabilized with $0.1 \%$ Triton X-100. Then the cells were incubated with following primary antibodies: goat anti-Nlrp3 (Abcam, ab4207) and mouse anti-caspase-1 (Santa cruz, SC-56036) respectively. After incubation with primary antibodies, the dishes were washed and labeled corresponding Alexa Fluor-488 (Invitrogen, 1827671) and Alexa Fluor-555 (CST, 4409S) conjugated secondary antibodies. Then the dishes were washed and visualized through sequentially scanning on an Olympus laser scanning confocal microscope (Fluoview FV1000, Olympus, Japan). Co-localization was analyzed by Image-Pro Plus software, and the co-localization was represented by Pearson's correlation coefficient.

\section{NADP+/NADPH assay kit}

The NADP ${ }^{+} / \mathrm{NADPH}$ assay in tissues or cells was tested by the commercial kit according to the manufacture's protocol (Beyotime Biotech, S0179). For the extraction of $\mathrm{NADP}^{+}$and NADPH, the renal tissues or cells was lysed and mixed with $400 \mu \mathrm{l}$ of extraction buffer. After centrifugation $\left(12,000 \times g, 10 \mathrm{~min}, 4^{\circ} \mathrm{C}\right)$ the supernatant was collected and kept on ice in the dark. To assess the total $\mathrm{NADP}^{+} / \mathrm{NADPH}$ content (NADPtotal), $50 \mu \mathrm{l}$ above supernatant of each sample was mixed with $100 \mu \mathrm{l}$ of $10 \mathrm{mM}$ G6-P and incubated for $10 \mathrm{~min}$. Then the product of formazan was determined by a maximum absorbance at $450 \mathrm{~nm}$ (Synergy HTX multifunction, enzyme marker, Vermont, USA). To test the NADPH content, $100 \mu \mathrm{l}$ of above supernatant of each sample was incubated in the water bath at $60^{\circ} \mathrm{C}$ for $30 \mathrm{~min}$ since heating destroys the oxidized form of NADP (i.e., NADP+) but has no effect on the reduced form (i.e., NADPH). After heating, $50 \mu \mathrm{l}$ supernatant of each sample was mixed with $100 \mu \mathrm{l}$ of $10 \mathrm{mM}$ G6-P and incubated for $10 \mathrm{~min}$. The absorbance at $450 \mathrm{~nm}$ was used to determine the NADPH content. The concentration of NADPtotal and NADPH was calculated according to the standard curve respectively. The formula as followed was used to evaluate $\mathrm{NADP}^{+} / \mathrm{NADPH}$ Assay.

$\left[\mathrm{NADP}^{+}\right]=[\mathrm{NADPtotal}]-[\mathrm{NADPH}]$,

$\left[\mathrm{NADP}^{+}\right] /[\mathrm{NADPH}]=([\mathrm{NADPtotal}]-[\mathrm{NADPH}]) /$ [NADPH].

\section{Reactive oxygen species ROS}

The ROS production in cells was determined by a commercial kit (Nanjing Jiancheng, E004-1-1). As described in the manufacture's protocol, the cells of each sample were incubated at $37^{\circ} \mathrm{C}$ for $1 \mathrm{~h}$ in the serum free medium containing DCFH-DA (1:1000) (Nanjing Jiancheng, E004-1-1). Then the cells were digested by trypsin (Gibco), washed by PBS for 2-3 times, and suspended in PBS. The fluorescence signals were detected at the excitation wavelength $485 \mathrm{~nm}$ and the emission wavelength $525 \mathrm{~nm}$ (Synergy HTX multifunction).

\section{CRISPR/Cas9 gene editing of APJ in HK2 cells}

Single-guide RNA sequence (gRNA) for CRISPR/Cas9 gene editing of protein-coding genes were designed by the CRISPR Design tool (http://crispr.mit.edu/). APJ gRNA 
sequences were CACCGCACAGACTGGAAATCCTCGG (sense) and AAACCCGAGGATTTCCAGTCTGTG (antisense). APJ gRNA sequences were synthesized and then inserted into the BbsI site of the plasmid pX459, generating pWHAPJg.

Gene editing in HK2 cells was carried out with Lipofectamine 3000 transfection (Invitrogen, L3000008) according to the manufacturer's guidelines. Briefly, a master mix of pX459 or pWHAPJg was mixed with P3000 and Lipofectamine 3000 reagent in the serum free medium and incubated at room temperature for $15 \mathrm{~min}$. Then the mixture was dropped into the wells and mixed softly and completely. After $24 \mathrm{~h}$, the transfected cells were incubated with $3 \mu \mathrm{g} / \mathrm{ml}$ puromycin (Sigma, P8833) in the media to screen out the pWHAPJg-containing cells. The gene-editing cells were selected from diluted single clones and the APJ knockout was confirmed by PCR.

\section{Statistical analysis}

Data are summarized as mean \pm stand error (SE). Statistical analysis was performed using ANOVA followed by a Student-Newman-Keuls post hoc test for multiple comparisons or by unpaired Student $t$ test for two comparisons. A $P$ value $<0.05$ was considered statistically significant.

\section{Results}

\section{Effect of ELA overexpression on the DOCA/salt-induced hypertension}

In DOCA/salt-treated rats, the mRNA level of ELA was found around $50 \%$ lower in the renal medulla (Fig. 1a). To explore the role of ELA on salt-sensitive hypertension, we performed intrarenal transfection of the ELA overexpression plasmid in the kidney of DOCA/salt hypertensive rats (Fig. 1b). Immunohistochemical staining for the overexpressed ELA-his tag with specific primary antibody of his-tag demonstrated an abundant accumulation of positive staining in both renal cortex and medulla in the DOCA/salt-treated rats, especially in the renal tubules (Fig. 1c). No obvious brown staining was detected in the kidneys without the ELA overexpressed vector transfection. These results verified a successful transfection and overexpression of ELA in kidney.

After 3-week treatment by DOCA/salt, the DOCA/saltsuppressed mRNA level of ELA was remarkably rescued by ELA overexpression in renal cortex and medulla (Fig. 1d). As shown in Fig. 2a-c, the systolic blood pressure (SBP), diastolic blood pressure (DBP), and mean arterial pressure (MAP) were significantly increased in the DOCA/salt group. The DOCA/salt-induced increase of BP was substantially attenuated by the renal ELA overexpression but still a little higher than that of control group (Fig. 2a-c). These results demonstrated that renal ELA had an antihypertensive effect in the DOCA/salttreated rats.

\section{Effects of ELA overexpression on glomerular injury and renal fibrosis in DOCA/salt-treated rats}

As shown in Fig. 2d, histologic analysis showed that rats in DOCA/salt group exhibited abnormal glomerular morphology implicated by the higher glomerular damage index compared with control group. However, the morphological damage was diminished in ELA treatment group. Moreover, the DOCA/salt treatment caused a dramatic elevation of the blood urea nitrogen (BUN) levels, which was significantly attenuated by ELA treatment (Fig. 2e). Similarly, serum IL-1 $\beta$ induced by the DOCA/salt treatment was decreased by ELA (Fig. 2f).

The upregulated expression of collagen and $\alpha$-SMA is always used as the index of renal fibrotic injury. Thus the collagen and $\alpha$-SMA levels were determined in the kidneys of these three groups. First, the Picro-sirius red was used to detect the total collagen. As shown in Fig. 3a, b, the positive-stained area of collagen was greatly enhanced in the DOCA/salt-treated kidneys than those in the control ones, which was diminished in the rats treated with ELA overexpression. Then the $\alpha$-SMA level was determined by immunochemistry and the results showed that DOCA/salt remarkably increased brown staining of $\alpha$-SMA in kidney but this increase was attenuated by ELA overexpression (Fig. 3c, d). Consistently, the protein levels of the collagen protein subtypes I (collagen I) and $\alpha$-SMA in the renal cortex and medulla were elevated in the DOCA/salt-treated group but suppressed by ELA administration (Fig. 3e). Taken together, these data elucidated that renal ELA exerted beneficial effects on glomerular injury and renal fibrosis in DOCA/salt-induced hypertensive rats.

\section{Effects of ELA overexpression on the inhibition of NADPH oxidase/ROS/NLRP3 inflammasome pathway in the kidneys of DOCA/salt-induced hypertensive rats}

The mRNA and protein levels of NLRP3 in both renal cortex and medulla were significantly increased in the DOCA/salt-treated group compared to that in control group, which were dramatically inhibited by ELA (Fig. 4a, b). The same change pattern on cleaved Caspase- 1 was received: compared to that in control kidneys, the increased protein level of cleaved Caspase- 1 stimulated by DOCA/salt was remarkably suppressed in the DOCA/ salt-ELA treated kidneys (Fig. 4a). IL-1 $\beta$, IL-18, and MCP1 are known as the downstream inflammatory markers of NLRP3 inflammasome. Thus the expression of IL-1 $\beta$, IL18 , and MCP-1 were assessed. The results showed that the stimulation of these three inflammatory markers by DOCA/salt treatment was notably blunted by ELA both in the renal cortex and medulla (Fig. 4c). These data demonstrated that ELA overexpression inhibited the expression and activation of NLRP3 inflammasome in the kidneys of DOCA/salt-induced hypertensive rats. 


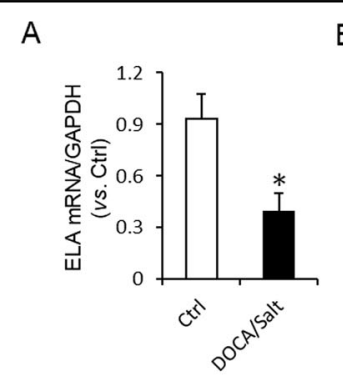

C

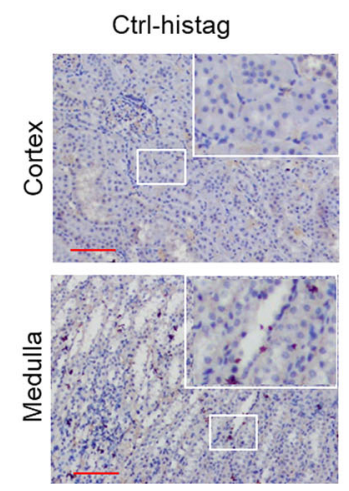

B

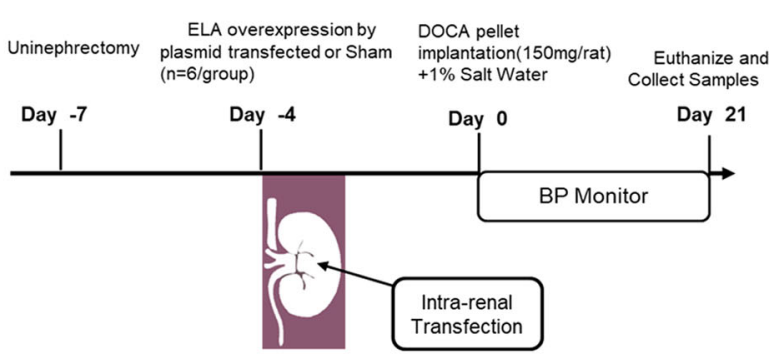

D
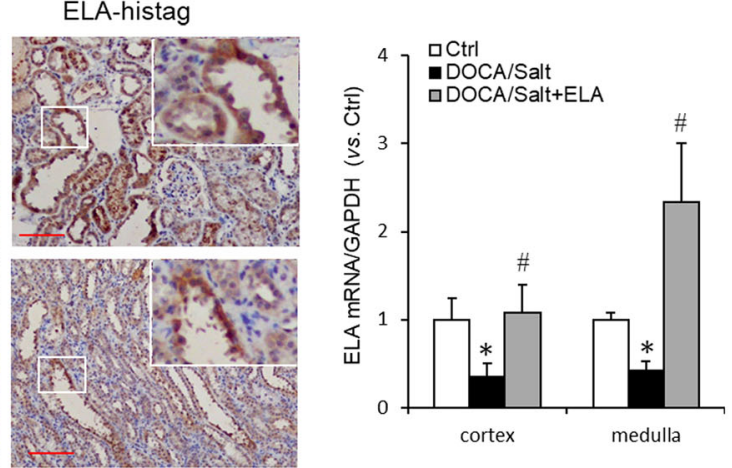

Fig. 1 Expression and rescue experiment of ELA in DOCA/salt-induced hypertensive rats. a The mRNA level of ELA in the renal medulla of DOCA/salt-treated rats ( $n=6$ per group). b Diagram of the animal protocol for ELA treatment in this study. c Representative immunohistochemical staining of ELA-histag in renal cortex and medulla. Kidneys without ELA-his tag transfection were used for Negative control. Scale bar, $100 \mu \mathrm{m}$. $\mathbf{d}$ The mRNA level of ELA in the kidneys of control group, DOCA/salt-treated group and DOCA/salt + ELA treated group $\left(n=6\right.$ per group). ${ }^{*} P<0.05$ versus Control group; ${ }^{\# P}<0.05$ versus DOCA/salt group.

Previous studies show that NADPH oxidase donates an electron from NADPH to molecular oxygen $\left(\mathrm{O}_{2}\right)$ to produce a superoxide anion $\left(\mathrm{O}_{2}{ }^{-}\right)^{34}$, which is a stimulator of NLRP3 inflammasome. The activation of NADPH oxidase is dependent on the assembly of subunits, such as $\mathrm{p} 22^{\text {phox }}$, gp91 ${ }^{\text {phox }}, \mathrm{p} 47^{\text {phox }}$, and $\mathrm{p} 67^{\text {phox } 17}$. Hence, we determined the mRNA expression of $\mathrm{p} 22^{\text {phox }}, \mathrm{gp} 91^{\text {phox }}, \mathrm{p} 47^{\text {phox }}$, and p $67^{\text {phox }}$ in both renal cortex and medulla first. In DOCA/ salt-treated group, the mRNA expression of $\mathrm{p} 22^{\text {phox }}$, gp91 ${ }^{\text {phox }}, \mathrm{p} 47^{\text {phox }}$, and $\mathrm{p} 67^{\text {phox }}$ were greatly elevated (Fig. $5 a)$ in renal cortex, indicating enhanced NADPH oxidase formation. Such elevation was attenuated by ELA administration (Fig. 5a). In renal medulla, the expression patterns of $\mathrm{p} 22^{\text {phox }}$ and $\mathrm{p} 47^{\text {phox }}$ were similar to that in the renal cortex; however no significant expression changes were detected for $\mathrm{gp} 91^{\mathrm{phox}}$ and $\mathrm{p} 67^{\mathrm{phox}}$ in these three animal groups (Fig. 5b). Consistently, the enhanced protein levels of $\mathrm{p} 22^{\text {phox }}$ and $\mathrm{p} 47^{\text {phox }}$ by DOCA/salt were diminished by ELA overexpression (Fig. 5c).

Moreover, NADPH is the major subcellular provider of reducing equivalents, which can be oxidized to $\mathrm{NADP}^{+}$. Thus the ratio of $\mathrm{NADP}^{+}$to $\mathrm{NADPH}\left(\mathrm{NADP}^{+} / \mathrm{NADPH}\right)$ is usually used to reflect the NADPH oxidase activation and subsequently ROS production ${ }^{35}$. As shown in Fig. 5d, the ratio of $\mathrm{NADP}^{+} / \mathrm{NADPH}$ was significantly increased in DOCA/salt-treated group, indicating the activation of NADPH oxidase and raised ROS production. And such increase was obviously alleviated by ELA in kidney.

Taken together, the data above suggested that renal ELA exerted suppressing effects on the NADPH oxidase/ ROS/NLRP3 inflammasome pathway in the DOCA/saltinduced hypertension.

\section{Effects of ELA on the NADPH oxidase/ROS/NLRP3 inflammasome pathway induced by Aldosterone in HK2 cells}

To further confirm the suppressing effects of ELA on the NADPH oxidase/ROS/NLRP3 inflammasome pathway, a series of tests were assessed in HK2 cells after the treatment of Aldosterone (Aldo) with or without ELA peptide. First, we determined the effect of ELA on the formation and activation of NLRP3 inflammasome induced by Aldo in HK2 cells. Aldo treatment resulted in strengthened co-localization (yellow spots) of NLRP3 molecules (green) with Caspase-1 (red) in HK2 cells compared to that of control cells, indicating enhanced formation of NLRP3 inflammasome (Fig. 6a). Unsurprisingly, such strengthened co-localization was significantly 


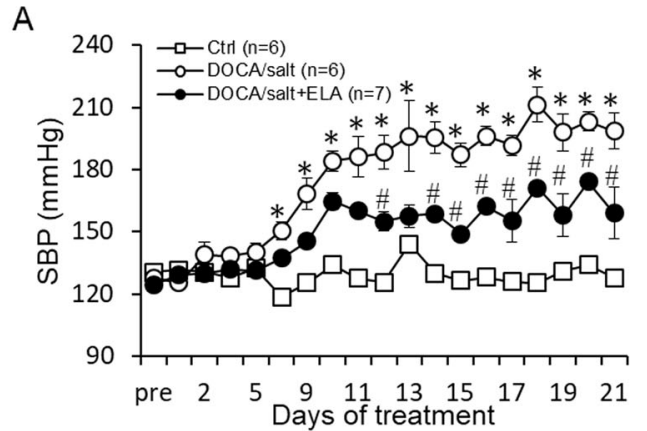

B

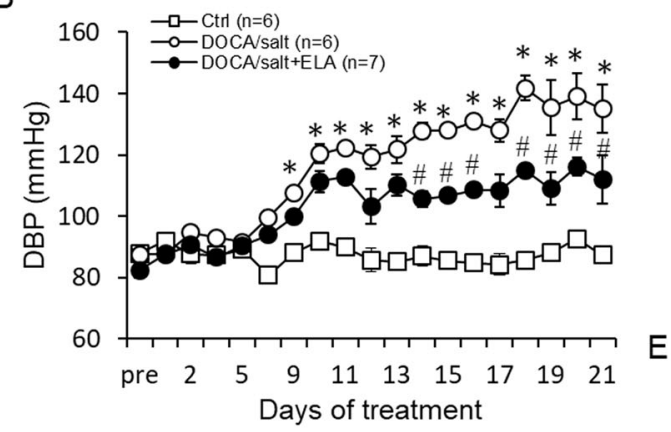

C

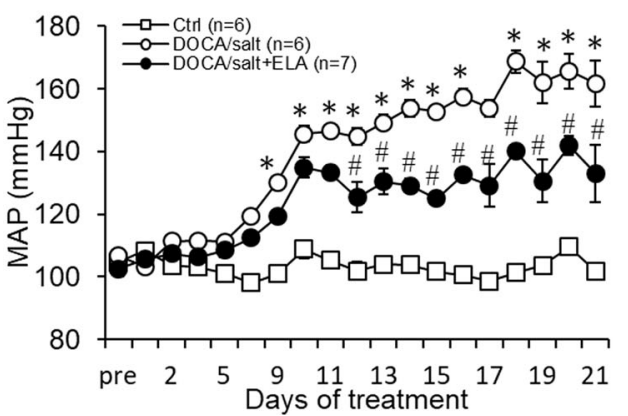

D
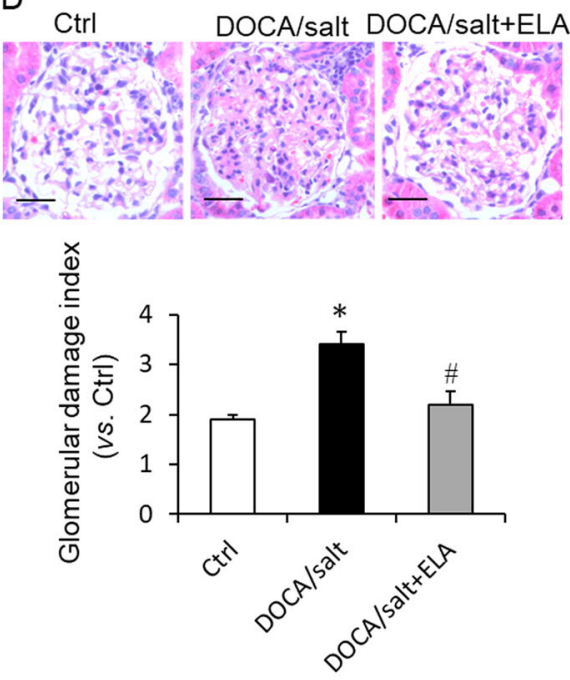

$E$
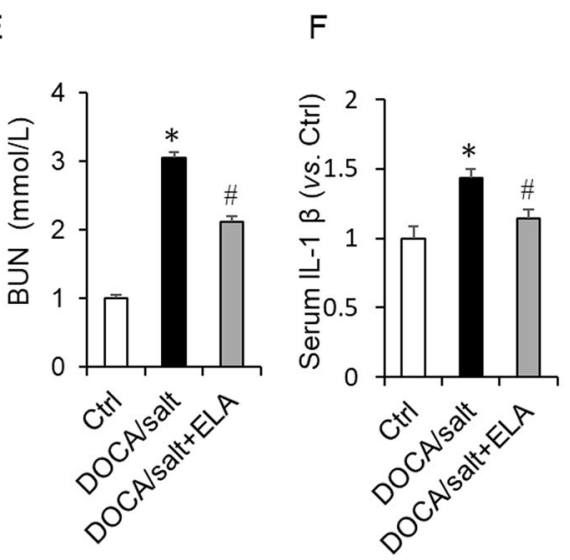

Fig. 2 Effects of ELA on DOCA/salt-induced increase in blood pressure, serum BUN, and IL-1 $\boldsymbol{\beta}$. a Systolic blood pressure (SBP). b Diastolic blood pressure (DBP). c Mean arterial pressure (MAP). d Representative photomicrographs showing glomerular structures (hematoxylin-eosin staining, 400X) and summarized glomerular damage index in different groups. Scale bar, $25 \mu \mathrm{m}$. e Serum blood urea nitrogen (BUN). f Serum interleukin-1 $\beta$ (IL-1 $\beta) . N=6$ per group. ${ }^{*} P<0.05$ versus Control group; ${ }^{\#} P<0.05$ versus DOCA/salt group. BUN blood urea nitrogen, IL-1 $\beta$ interleukin-1 $\beta$.

attenuated by ELA peptide (Fig. 6a). The co-localization coefficient analyses were summarized in Fig. 6b. Consistently, Aldo-induced Caspase-1 cleavage was inhibited by ELA (Fig. 6c). Furthermore, the increased ROS production (Fig. 6d) and the enhanced ratio of NADP $+/ \mathrm{NADPH}$ (Fig. 6e) were observed in Aldo treated cells, which were markedly alleviated by ELA pretreatment. No significant difference was detected on the co-localization of NLRP3 with caspase-1, the protein level of cleaved Caspase-1, ROS production and the ratio of NADP $+/ \mathrm{NADPH}$ in cells only treated with ELA treatment or not. These results clearly suggested that ELA had inhibitory effects on the Aldosterone-induced NADPH oxidase/ ROS/NLRP3 inflammasome in HK2 cells.
Effects of ELA in the DOCA/salt-induced hypertension are APJ independent

ELA is reported to be an endogenous ligand for apelin receptor (APJ) and regulates fluid homeostasis by binding to APJ in kidney ${ }^{26}$. And APJ has been found involved in the regulation of NADPH oxidase and ROS production $^{36,37}$. Thus, we examined whether ELA was dependent on APJ to inhibit NADPH oxidase/ROS/NLRP3 inflammasome pathway in the DOCA/salt-treated kidneys. As shown in Fig. 7a, the protein level of APJ was dramatically inhibited both in renal cortex and medulla of the DOCA/ salt-treated rats. ELA overexpression was unable to recover the inhibition of APJ by DOCA/salt (Fig. 7a), implying that the antihypertensive effect of ELA may be 

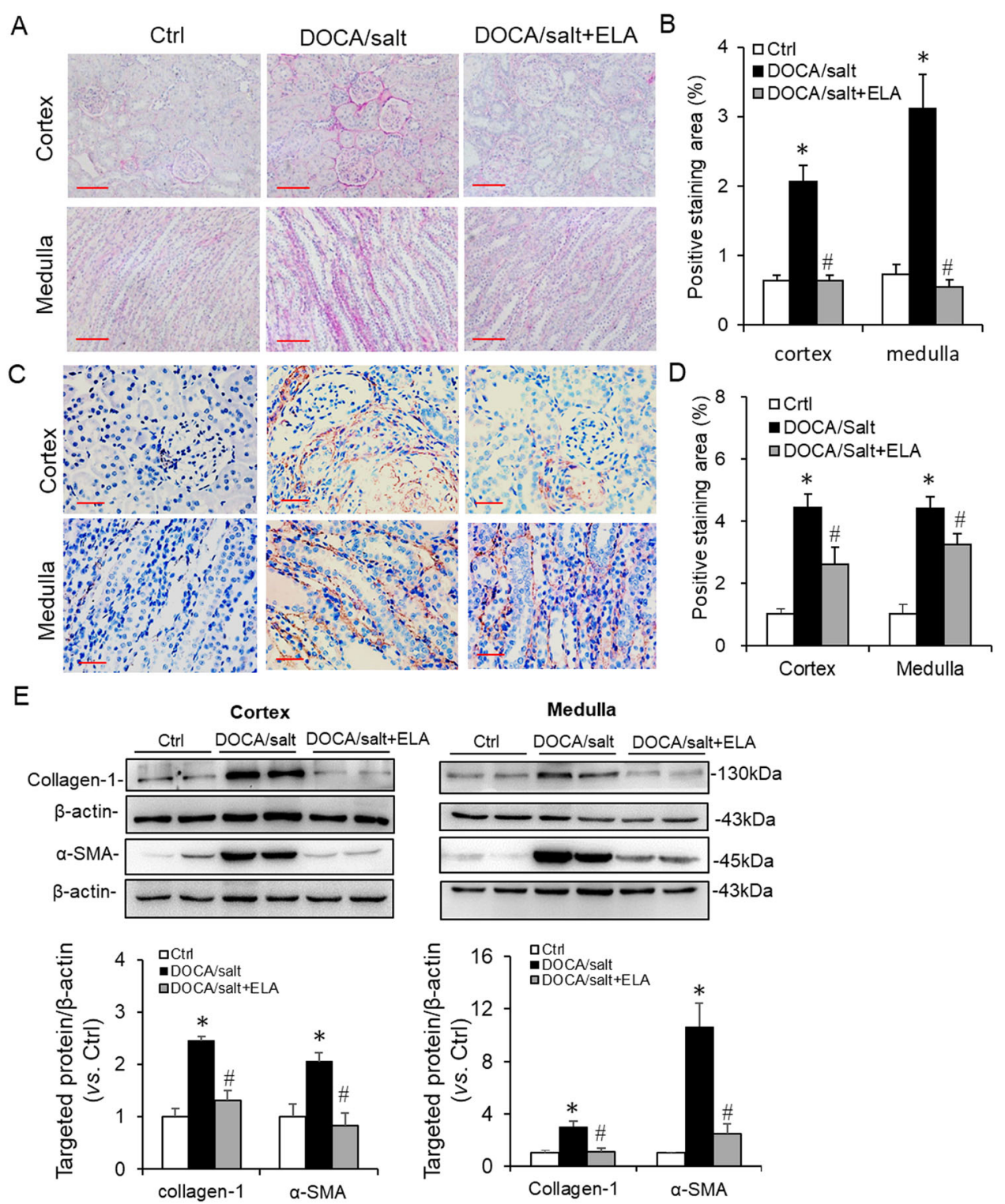

Fig. 3 Effects of ELA on DOCA/salt-induced renal fibrosis. a Representative Picro-sirius red staining of collagen in renal cortex and medulla. Scale bar, $100 \mu \mathrm{m}$. b Summarized Picro-sirius red staining in renal cortex and medulla. c Representative immunohistochemical staining of a-SMA in renal cortex and medulla. Scale bar, 50 mm. d Summarized immunohistochemical staining in renal cortex and medulla. e Representative immunoblots of Collagen-1 and a-SMA in renal cortex and medulla as well as summarized intensities of blots. $N=6$ per group. ${ }^{*} P<0.05$ versus $C$ trl group; ${ }^{\#} P<0.05$ versus DOCA/salt group. a-SMA a-smooth muscle actin.

independent on APJ in vivo. To further investigate whether APJ is involved in the inhibitory effects of ELA on Aldo-induced NADPH oxidase/ROS/NLRP3 inflammasome pathway, APJ was knocked down (KD) in HK2 cells, which was verified by real-time PCR (Fig. 7b). Interestingly, Aldo-induced ROS production was still suppressed by ELA in the APJ KD cells (Fig. 7c). Consistently, ELA peptides blocked the enhanced co-localization (yellow spots) of NLRP3 molecules (green) with caspase-1 (red) stimulated by Aldo in APJ KD cells (Fig. 7d). The colocalization coefficient analyses were summarized in Fig. 7e. No statistical significance was detected on the ROS production or co-localization of NLRP3 molecules (green) with caspase-1 (red) in APJ KD cells only treated with ELA treatment or not. Thus, our in vivo and in vitro results suggested that the protective roles of ELA in the DOCA/salt-induced hypertension are independent on APJ in the kidney. 


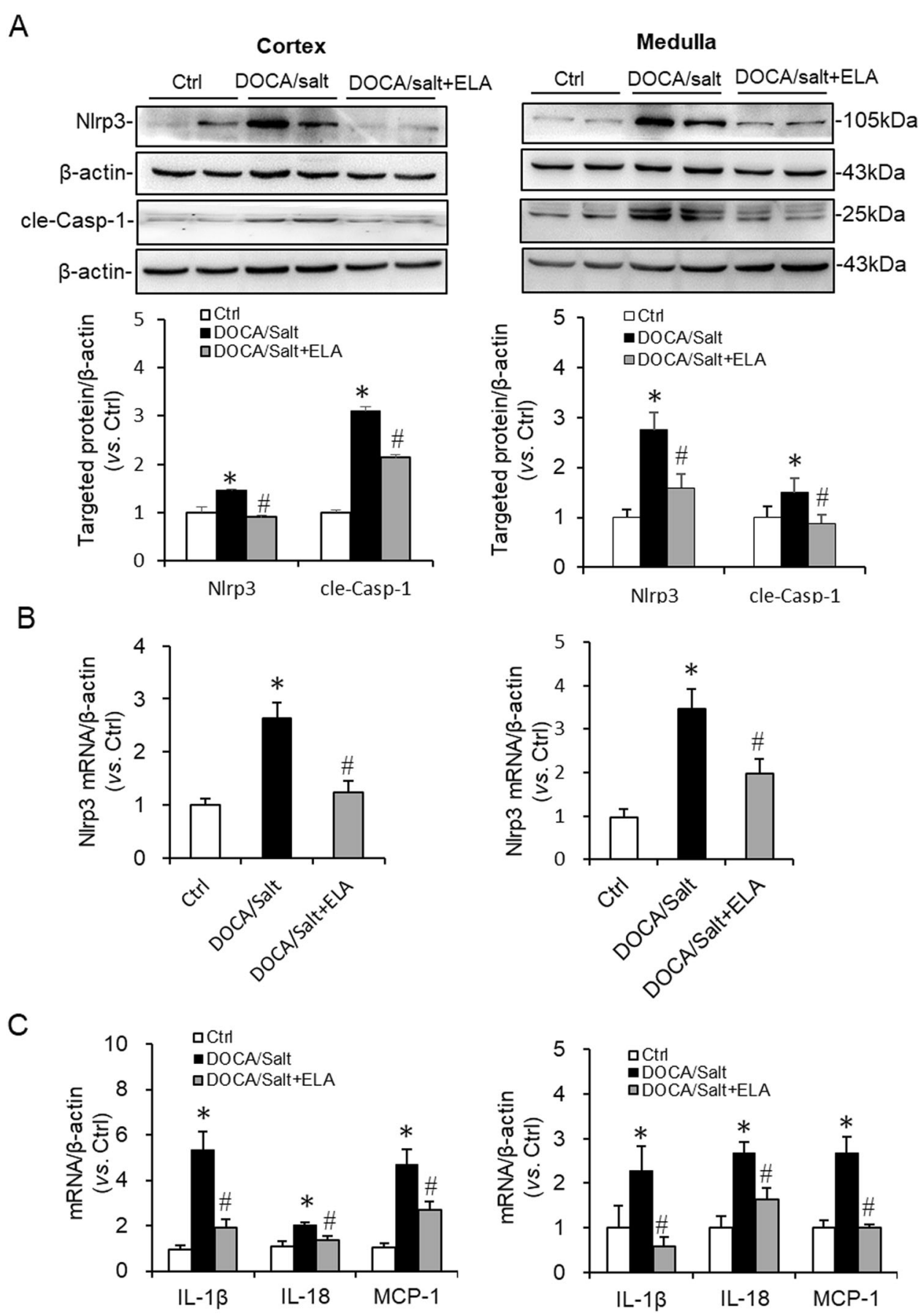

Fig. 4 Effects of ELA on the DOCA/salt-induced activation of NLRP3 inflammasome. a Representative immunoblots of NLRP3 and cle-Casp-1 in renal cortex and medulla as well as summarized intensities of blots. $\mathbf{b}$ NLRP3 mRNA levels in the renal cortex and medulla. $\mathbf{c}$ mRNA levels of IL-1 $\beta$, IL-18, and MCP-1 in the renal cortex and medulla. $N=6$ per group. ${ }^{*} P<0.05$ versus Ctrl group; ${ }^{\#} P<0.05$ versus DOCA/Salt group. NLRP3 Nod-like receptor protein 3, cle-Casp-1 cleaved Caspase-1, IL-18 Interleukin-1 $\beta$, MCP-1 monocyte chemotactic protein 1.

ELA deficiency accelerated the onset of hypertension in DOCA/salt hypertensive mice

To further confirm the antihypertensive effect of ELA, the $\mathrm{ELA}^{+/-}$mice were used to observe the change of BP with DOCA/salt treatment. The mRNA expression of
ELA was about $70 \%$ decreased in the kidney of the ELA $^{+/-}$mice compared to that of WT mice (Fig. 8a), suggesting a successful knockdown of ELA. As shown in Fig. 8b, DOCA/salt treatment progressively increased the SBP in the $\mathrm{ELA}^{+/-}$and WT mice, while the BP remained 

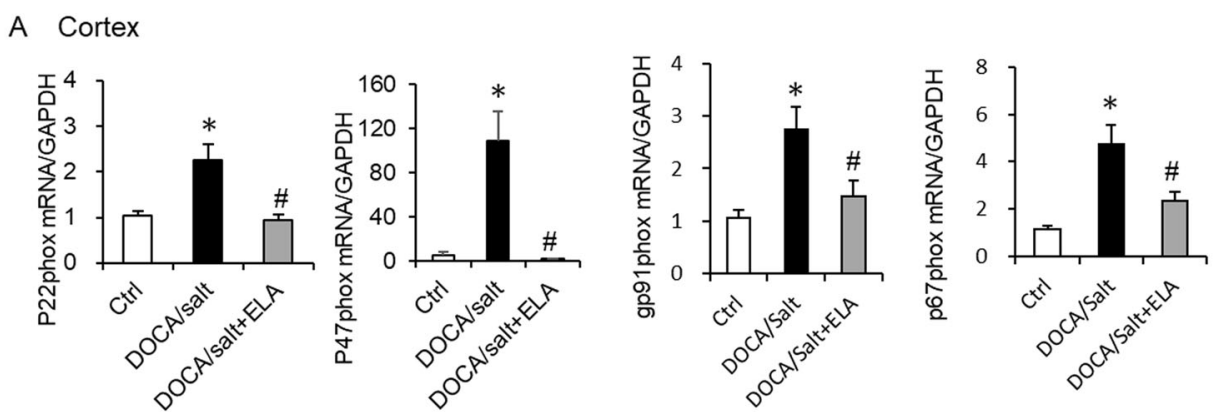

B Medulla
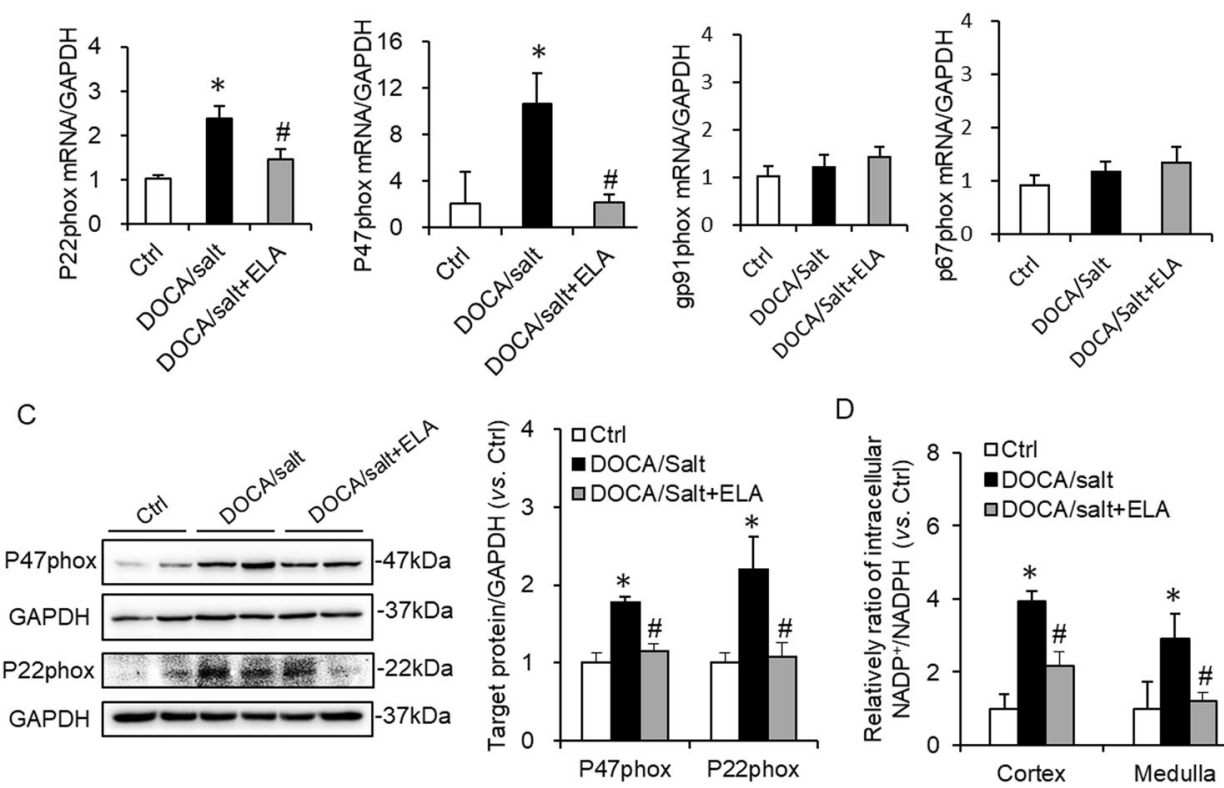

D

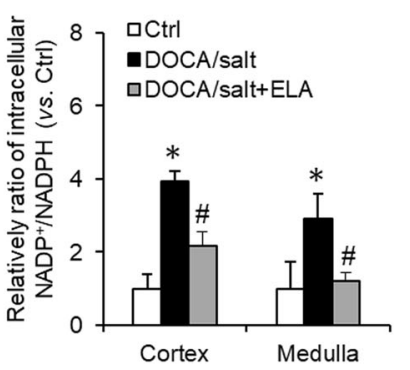

Fig. 5 Effects of ELA on DOCA/salt-induced NADPH oxidase formation and activation. a mRNA levels of p22 ${ }^{\text {phox }}, g^{p} 91^{\text {phox }}, \mathrm{p} 47^{\text {phox }}$, and p6 $7^{\text {phox }}$ subunits of NADPH oxidase in the renal cortex. $\mathbf{b}$ mRNA levels of $\mathrm{p} 22^{\text {phox }}, \mathrm{gp} 91^{\text {phox }}, \mathrm{p} 47^{\text {phox }}$, and $\mathrm{p} 67^{\text {phox }}$ subunits of NADPH oxidase in the renal medulla. $\mathbf{c}$ Representative immunoblots of $\mathrm{p} 22^{\text {phox }}$ and $\mathrm{p} 47^{\text {phox }}$ and summarized intensities of blots. $\mathbf{d}$ Intracellular NADP ${ }^{+} / \mathrm{NADPH}$ in renal cortex and medulla. $N=6$ per group. ${ }^{*} P<0.05$ versus Ctrl group; ${ }^{\#} P<0.05$ versus DOCA/salt group.

unchanged in the WT control and $\mathrm{ELA}^{+/-}$control animals. More interestingly, compared to WT mice, $\mathrm{ELA}^{+/-}$ mice exhibited an earlier increase of SBP in response to the DOCA/salt treatment, especially in the first 2 weeks (Fig. 8b). The SBP of ELA ${ }^{+/-}$and WT mice treated with DOCA/salt at the 14th day were $156 \pm 7 \mathrm{mmHg}$ and $120 \pm 4 \mathrm{mmHg}$ respectively. At the end of the animal experiment, the SBP of the ELA ${ }^{+/-}$DOCA/salt group $(146 \pm 3 \mathrm{mmHg})$ was still a little higher than that of the WT DOCA/salt group $(134 \pm 2 \mathrm{mmHg})$ but no statistical significance existed between WT control group and ELA $^{+/-}$control group $(112 \pm 2 \mathrm{mmHg}$ vs. $108 \pm$ $2 \mathrm{mmHg}$ ). These results above suggested that ELA deficiency dramatically accelerated the onset of hypertension in DOCA/salt-treated mice, supporting a protective role of ELA on salt-sensitive hypertension.

\section{Discussion}

In the present study, we investigated the roles of ELA in DOCA/salt-induced hypertension and the potential mechanism. In DOCA/salt-treated rats, the mRNA level of ELA greatly decreased in the renal medulla (Fig. 1a). Then we found that overexpression of ELA in the kidney attenuated DOCA/salt-induced hypertension and renal injury, including lower BP, reversed glomerular morphological change, decreased serum BUN, and blocked fibrotic accumulation (Figs. 2, 3). Mechanistically, ELA overexpression inhibited renal NADPH oxidase activity and subsequent ROS production, thus resulted in the blockade of formation and activation of NLRP3 inflammasome stimulated by DOCA/salt (Figs. 4, 5). The inhibitory effects of ELA on Aldosterone-stimulated NADPH oxidase/ROS/NLRP3 inflammasome signaling pathway 
A
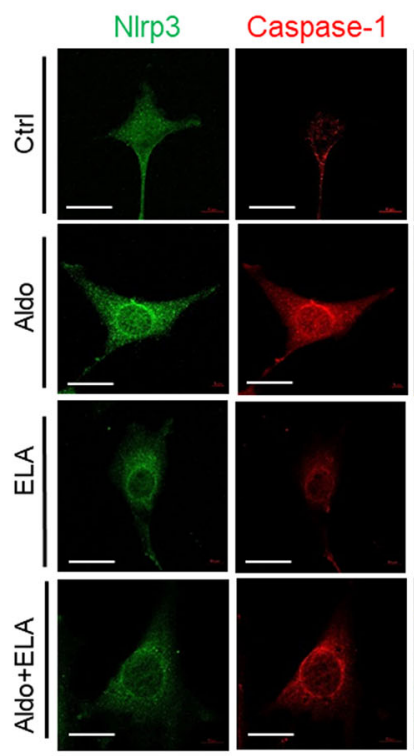

C
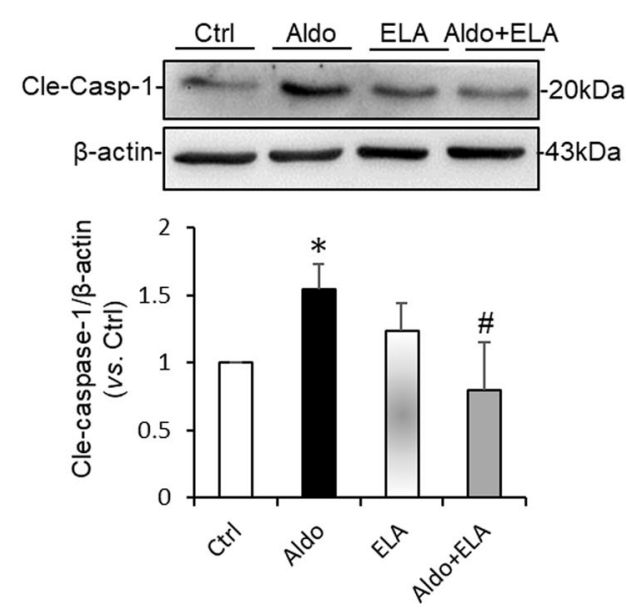

B

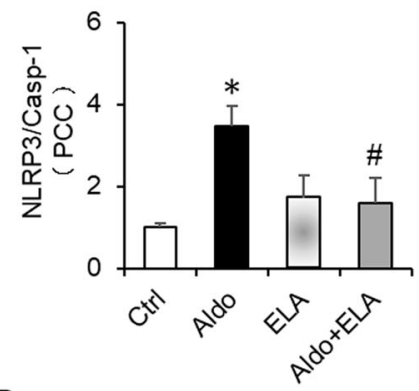

D
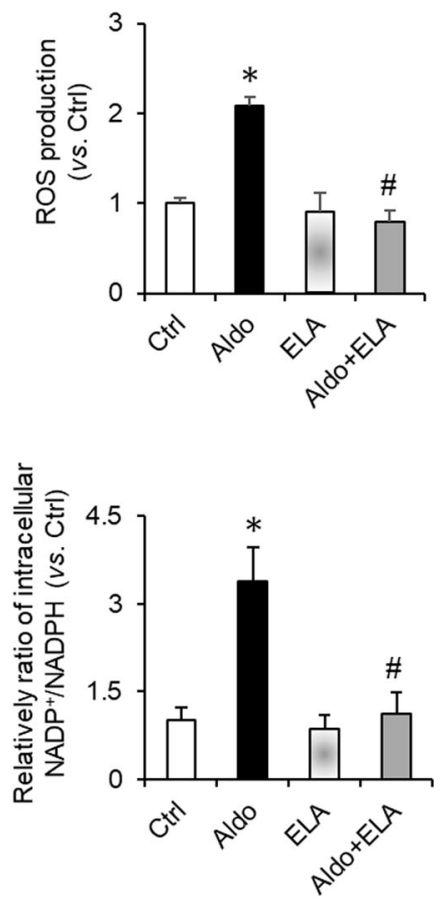

Fig. 6 Effects of ELA on Aldosterone-induced NADPH oxidase/ROS/NLRP3 pathway in HK2 cells. The HK2 cells were pretreated with ELA peptide $(1 \mathrm{nM})$ for $1 \mathrm{~h}$ and then treated by Aldosterone $(0.1 \mu \mathrm{M})$ for $24 \mathrm{~h}$. $\mathbf{a}, \mathbf{b}$ representative confocal fluorescent images of co-localization (yellow) between Nlrp3 (green)/Caspase-1 (red), Nlrp3 (green)/ASC (red) and summarized co-localization coefficient ( $n=5$ per group). Scale bar, $10 \mu m$. c Representative immunoblots of cleaved Caspase-1 and summarized intensities of blots ( $n=5$ per group). $\mathbf{d}$ ROS production ( $n=6$ per group). e Intracellular $\mathrm{NADP}^{+} / \mathrm{NADPH}$ ( $n=6$ per group). ${ }^{*} P<0.05$ versus $\mathrm{Ctrl}$ group; ${ }^{*} P<0.05$ versus DOCA/salt group. ROS reactive oxygen species, NADPH nicotinamide adenine dinucleotide phosphate.

was further confirmed in the HK2 cells (Fig. 6). Furthermore, our in vivo and in vitro results showed that the deficiency of the apelin receptor APJ did not influence on the antihypertensive effect and blockage to NADPH oxidase/ROS/NLRP3 pathway of ELA (Fig. 7). Additionally, in heterozygous ELA knockout mice $\left(\mathrm{ELA}^{+/-}\right)$, we found that ELA deficiency significantly accelerated the onset of DOCA/salt-induced hypertension (Fig. 8a, b). Taken together, our data suggest that renal ELA exerts its beneficial roles in DOCA/salt-induced hypertension via blocking NADPH oxidase/ROS/NLRP3 inflammasome pathway, which is independent on its endogenous receptor APJ. The potential mechanism of ELA functions on DOCA/salt-induced hypertension and renal injury was shown in Fig. 8c.

Previous studies show that ELA is expressed not only in embryos but also in adult kidneys ${ }^{24,29}$. In humans, a 54amino-acid (aa) full length of ELA bears a cleavage to yield a secretory mature form comprised of $32 \mathrm{aa}^{23}$. The amino-acid sequence analysis reveals multiple dibasic sites in the 32-aa ELA (ELA-32), implying more shorten isoforms existing in vivo ${ }^{38}$. Among these isoforms, an 


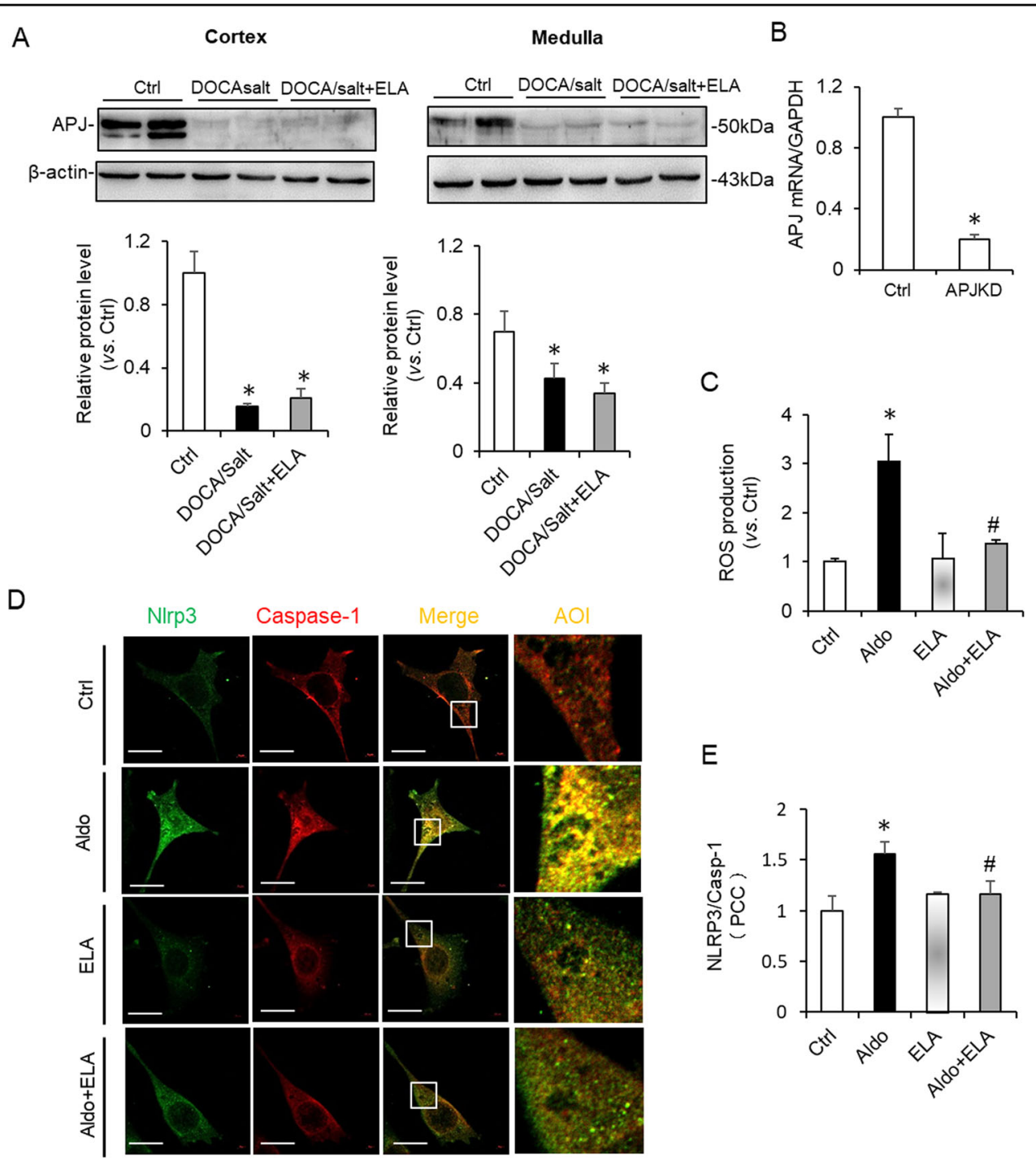

Fig. 7 Effects of ELA on the ROS production and NLRP3 imflammasome activation are independent of APJ. APJ knockdown was conducted by the transfection of plasmid pWHAPJg into HK2 cells and confirmed by RT-PCR. Then the HK2 cells with APJ knockdown were pretreated with ELA peptide $(1 \mathrm{nM})$ for $1 \mathrm{~h}$ and then treated by Aldosterone $(0.1 \mu \mathrm{M})$ for $24 \mathrm{~h}$. a Representative immunoblots of APJ in renal cortex and medulla and summarized intensities of blots. $\mathbf{b}$ mRNA levels of APJ in HK2 cells with or without APJ knockdown. c ROS production in HK2 cells with APJ knockdown. d, e Representative confocal fluorescent images of co-localization (yellow) between Nlrp3 (green)/Caspase-1 (red) and summarized co-localization coefficient in HK2 cells with APJ knockdown. Scale bar, $10 \mu \mathrm{m} . \mathrm{N}=6$ per group. ${ }^{*} P<0.05$ versus Ctrl group; ${ }^{\# P}<0.05$ versus DOCA/ salt group.

11-aa ELA (ELA-11) is highly conserved and endogenously observed via mass spectrometry in embryos ${ }^{25}$. It has been recently reported that both ELA-32 and ELA-11 are able to significantly inhibit H/R-induced renal fibrosis, inflammation, renal tubular lesions, and renal dysfunction $^{27}$. They also report that ELA-11 but not ELA-32 has inhibitory effects on I/R-induced autophagy in the kidney. It implies that different isoforms of ELA may function under different mechanisms. Whether there is a major functional form existing is not yet known. In this work, we tried to overexpress ELA peptide in the kidney by a transfected plasmid. The synthesized full length of ELA may be cleaved into various active forms and secreted into the whole kidney. The immunohistochemical results showed that ELA peptides existed in both renal cortex and medulla, especially in the renal tubules (Fig. 2b), which is consistent to the previous studies ${ }^{27,28}$. However, there are still some limits in this work. First, since lacking commercial and specific antibody of ELA, the protein level couldn't be determined in kidneys under all the 

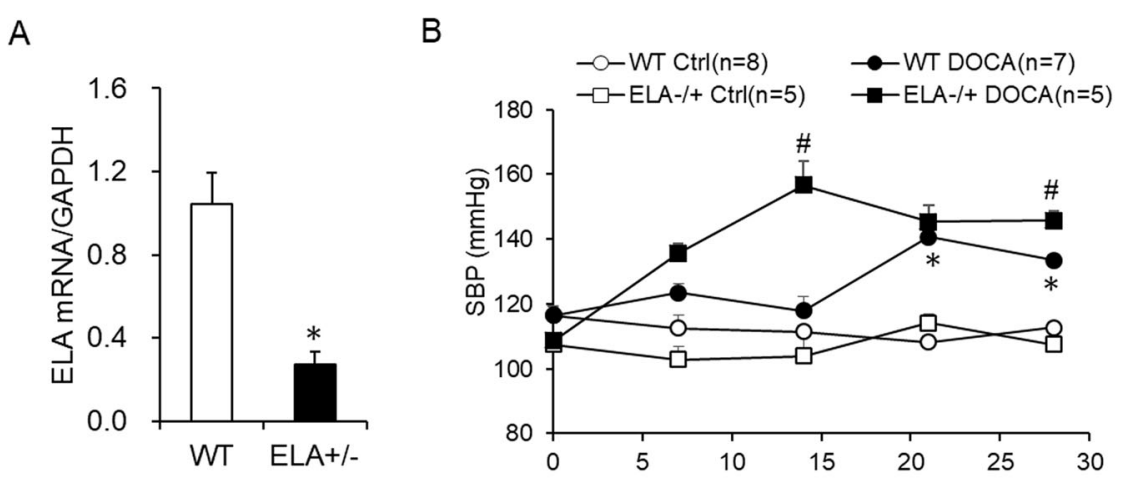

C

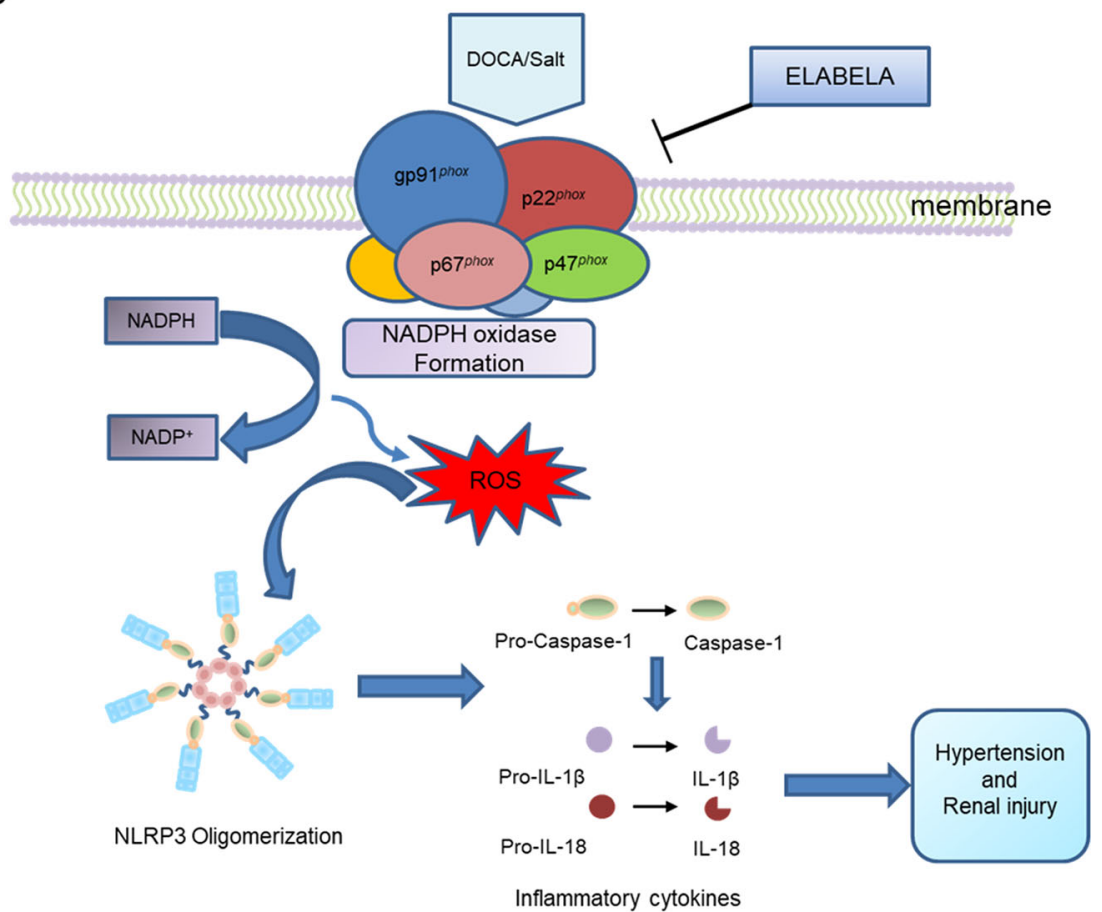

Fig. 8 Effects of ELA deficiency on DOCA/salt-induced hypertension and potential mechanism of ELA exerted on DOCA/salt-induced hypertension. a The mRNA level of ELA in the kidney of heterozygous ELA knockout C57BL/6 mice (ELA ${ }^{+-}$) compared to WT ones. $N=5$. b Systolic blood pressure (SBP). c Potential mechanism of ELA exerted on DOCA/salt-induced hypertension. In DOCA/salt-treated kidney, ELA blocks the NADPH oxidase formation by inhibiting the expression of its subunits $\mathrm{p} 22^{\text {phox }}$, gp9 ${ }^{\text {phox }}, \mathrm{p} 47^{\text {phox }}$, and p67 ${ }^{\text {phox }}$; decreases the ROS production; obstructs the recruitment and activation of NLRP3 inflammasome; attenuates hypertension and renal injury. ${ }^{*} P<0.05$ versus WT Ctrl group. ${ }^{\#} P<0.05$ versus DOCA/ salt-treated WT group.

experimental conditions. On the other hand, our results cannot tell different isoforms of ELA locally since the his tag was fused to the C terminus of ELA peptide.

Oxidative stress and inflammation are found to play essential roles in the occurrence and development of cardiaovascular diseases, chronic kidney diseases, diabetes with microvascular complications, tumors, and preeclampsia $^{39-42}$. Documents of studies show that ELA endogenous homologue apelin, through binding to its receptor APJ, is involved in the regulation of oxidative stress and inflammation ${ }^{43}$. However, whether the role of apelin is beneficial or aggravating remains controversial. For example, a previous study demonstrates that apelin reduces oxidative stress and prevents pressure-overloadinduced left ventricular hypertrophy ${ }^{44}$. By contrast, there is increasing evidence proving that apelin-13 induces cardiac hypertrophy by increasing the production of ROS and the expression levels of NADPH oxidases in vivo and in vitro $^{45-47}$. In addition, apelin/APJ in paraventricular nucleus is found to induce long-term high $\mathrm{BP}$ and renal sympathetic nerve activity via increasing oxidative stress $^{37,48}$. Thus, there is no identical conclusion on the 
association of apelin/APJ with oxidative stress and inflammation. Different from that on apelin, the related studies on ELA are limited. Our data demonstrated that ELA had inhibitory effects on NADPH oxidase/ROS/ NLRP3 pathway in the kidney to improve DOCA/saltinduced hypertension. Such effects of ELA peptide were also confirmed in the human renal tubular duct epithelial cells.

In addition, it is known that the regulatory effects of apelin on oxidative stress are conducted by binding to its receptor APJ in most experimental conditions ${ }^{37,46,47}$. As another endogenous ligand, it is still unclear whether ELA is dependent on APJ to suppress DOCA/salt-induced NADPH oxidase/ROS/NLRP3 signaling pathway. Our results in this work found that APJ was dramatically inhibited in both renal cortex and medulla in DOCA/saltinduced hypertensive rats and such inhibition was not rescued by overexpression of ELA. These indicated that APJ may have no effect on the improving roles of ELA in the DOCA/salt-treated kidney. Moreover, even as APJ was around $80 \%$ downregulated in HK2 cells, ELA still blocked the Aldo-induced ROS production and NLRP3 inflammasome formation. Thus, our results suggested that the antihypertensive effect and inhibition to NADPH oxidase/ROS/NLRP3 pathway of ELA does not rely on the $\mathrm{G}$ protein receptor APJ. It further raised an interesting question how ELA works on NADPH oxidase/ROS in DOCA/salt-treated rats, directly or indirectly. Similar phenomena are reported recently that ELA can function independently on $\mathrm{AP}^{27}$, although ELA is verified to directly bind to the APJ receptor with high affinity ${ }^{26}$. Little is known about the underlying mechanism. It is assumed by Chen et al. that higher dosages of ELA ( 0.3 and $3 \mathrm{mM})$ but not lower dose $(300 \mathrm{pM})$ could induce APJ endocytosis and the downstream phosphorylation of ERK to accomplish the reno-protective functions ${ }^{27}$. There is another possibility that other kind of receptor may exist to mediate the effects of ELA. Thus, it is of great interest to explore whether other potential receptors or a competitive mechanism is involved for further understanding the roles of ELA in the kidney.

There are several studies showing that exogenous treatment of ELA peptides results in protective and improving effects on kidney. Deng et al. find that intravenous injections of ELA peptide increase the urine flow rate and water intake and these effects are dose dependent $^{26}$. Another study suggests that ELA treatment protects the kidney from $I / R$ stress ${ }^{27}$. To fully understand the roles of ELA, the ELA knockout mouse is a useful tool. However, a recent study reveals that knockout of ELA leads to lethal cardiac defects in some ELA null embryos $^{49}$. Thus, heterozygous ELA knockout C57BL/6 mice $\left(\mathrm{ELA}^{+-}\right)$were used to verify its functions in this work. Compared to the WT mice, the expression of ELA in $\mathrm{ELA}^{+/-}$kidneys was about $70 \%$ lower (Fig. 8a), suggesting a successful ELA deficiency. We found that the $\mathrm{ELA}^{+/-}$mice presented no significant hypertension at the basic condition (Fig. 8b). However, ELA ${ }^{+/-}$mice exhibited an earlier increase of SBP than WT ones in response to the DOCA/salt treatment, especially in the first 2 weeks (Fig. 8b). At the end of the animal experiment, the $\mathrm{ELA}^{+/-}$DOCA/salt group still had a little higher of SBP than the WT DOCA/salt group. These results of BP suggested that ELA deficiency dramatically accelerated the onset of hypertension in DOCA/salt-induced hypertensive mice, further confirming a protective role of ELA on salt-sensitive hypertension.

\section{Conclusion}

In summary, the present study investigates the role of ELA in the development of DOCA/salt-induced hypertension and renal injury in rodent models. Our data demonstrate that ELA may prevent DOCA/salt-induced hypertension by blocking NADPH oxidase/ROS/NLRP3 signaling pathway in the kidney, which is APJ independent. All these provide new sight on ELA as a candidate to treat salt-sensitive hypertension and renal injury.

\section{Acknowledgements \\ This work was supported by National Natural Science Foundation of China Grant No. 81600322 and No. 81770707. \\ Author details \\ ${ }^{1}$ School of Pharmaceutical Sciences, Guangzhou University of Chinese Medicine, Guangzhou, China. ²Department of Nephrology, The Second Affiliated Hospital, School of Medicine, Zhejiang University, Hangzhou, China. ${ }^{3}$ Guangdong Metabolic Disease Research Center of Integrated Chinese and Western Medicine, Guangdong Pharmaceutical University, Guangzhou, China}

\section{Author contributions}

L.W., Q.Z., and Z.C. designed the experiments and drafted the paper. Z.C., C.W., C.H., and H.L. collected the animal samples and analyzed the effects of ELA by molecular methods and ELISA tests in tissues and cells. Z.C., C.W., Y.L., Y.Z., and C.H. did animal experiments and recorded the BP. H.L., Q.Q., and M.H. were in charge of the breeding, genotyping identification, and taking care of heterozygous ELA knockout C57BL/6 mice.

Conflict of interest

The authors declare that they have no conflict of interest.

\section{Publisher's note}

Springer Nature remains neutral with regard to jurisdictional claims in published maps and institutional affiliations.

Received: 28 February 2020 Revised: 5 August 2020 Accepted: 5 August 2020

Published online: 22 August 2020

\footnotetext{
References

1. O'Donnell, M., Mente, A. \& Yusuf, S. Sodium intake and cardiovascular health. Circ. Res. 116, 1046-1057 (2015).

2. McDonough, A. A. Mechanisms of proximal tubule sodium transport regulation that link extracellular fluid volume and blood pressure. Am. J. Physiol. Regul. Integr. Comp. Physiol. 298, R851-R861 (2010).
} 
3. Banek, C. T., Gauthier, M. M., Van Helden, D. A., Fink, G. D. \& Osborn, J. W. Rena inflammation in DOCA-salt hypertension. Hypertension 73, 1079-1086 (2019).

4. Wang, D. et al. Serelaxin improves cardiac and renal function in DOCA-salt hypertensive rats. Sci. Rep. 7, 9793 (2017).

5. Iyer, A., Chan, V. \& Brown, L. The DOCA-salt hypertensive rat as a model of cardiovascular oxidative and inflammatory stress. Curr. Cardiol. Rev. 6, 291-297 (2010).

6. McMaster, W. G., Kirabo, A., Madhur, M. S. \& Harrison, D. G. Inflammation, immunity, and hypertensive end-organ damage. Circ. Res. 116, 1022-1033 (2015).

7. Krishnan, S. M. et al. Inflammasome activity is essential for one kidney/deoxycorticosterone acetate/salt-induced hypertension in mice. Br. J. Pharmacol. 173, 752-765 (2016).

8. Latz, E. The inflammasomes: mechanisms of activation and function. Curr. Opin. Immunol. 22, 28-33 (2010).

9. Abais, J. M. et al. Contribution of endogenously produced reactive oxygen species to the activation of podocyte NLRP3 inflammasomes in hyperhomocysteinemia. Free Radic. Biol. Med. 67, 211-220 (2014).

10. Chen, J. \& Chen, Z. J. Ptdlns4P on dispersed trans-Golgi network mediates NLRP3 inflammasome activation. Nature 564, 71-76 (2018).

11. Krishnan, S. M. et al. Pharmacological inhibition of the NLRP3 inflammasome reduces blood pressure, renal damage, and dysfunction in salt-sensitive hypertension. Cardiovasc. Res. 115, 776-787 (2019).

12. Qi., J. et al. Targeting interleukin-1 beta to suppress sympathoexcitation in hypothalamic paraventricular nucleus in Dahl salt-sensitive hypertensive rats. Cardiovasc. Toxicol. 16, 298-306 (2016).

13. Zhang, C. et al. Activation of Nod-like receptor protein 3 inflammasomes turns on podocyte injury and glomerular sclerosis in hyperhomocysteinemia. Hypertension 60, 154-162 (2012).

14. Abais, J. M. et al. NADPH oxidase-mediated triggering of inflammasome activation in mouse podocytes and glomeruli during hyperhomocysteinemia. Antioxid. Redox Signal. 18, 1537-1548 (2013)

15. Gross, O., Thomas, C. J., Guarda, G. \& Tschopp, J. The inflammasome: an integrated view. Immunol. Rev. 243, 136-151 (2011).

16. Babior, B. M., Lambeth, J. D. \& Nauseef, W. The neutrophil NADPH oxidase. Arch. Biochem. Biophys. 397, 342-344 (2002).

17. Groemping, Y., Lapouge, K., Smerdon, S. J. \& Rittinger, K. Molecular basis of phosphorylation-induced activation of the NADPH oxidase. Cell 113, 343-355 (2003).

18. Lejal, N. et al. Turning off NADPH oxidase-2 by impeding p67(phox) activation in infected mouse macrophages reduced viral entry and inflammation. Biochim. Biophys. Acta Gen. Subj. 1862, 1263-1275 (2018).

19. Zhang, A., Jia, Z., Wang, N., Tidwell, T. J. \& Yang, T. Relative contributions of mitochondria and NADPH oxidase to deoxycorticosterone acetate-salt hypertension in mice. Kidney Int. 80, 51-60 (2011).

20. Beswick, R. A., Dorrance, A. M., Leite, R. \& Webb, R. C. NADH/NADPH oxidase and enhanced superoxide production in the mineralocorticoid hypertensive rat. Hypertension 38, 1107-1111 (2001).

21. Ding, $W$. et al. Mitochondrial reactive oxygen species-mediated NLRP3 inflammasome activation contributes to aldosterone-induced renal tubular cells injury. Oncotarget 7, 17479-17491 (2016).

22. Chng, S. C., Ho, L., Tian, J. \& Reversade, B. ELABELA: a hormone essential for heart development signals via the apelin receptor. Dev. Cell 27, 672-680 (2013).

23. Xu, J., Chen, L., Jiang, Z. \& Li, L. Biological functions of Elabela, a novel endogenous ligand of APJ receptor. J. Cell. Physiol. 233, 6472-6482 (2018).

24. Pauli, A. et al. Toddler: an embryonic signal that promotes cell movement via Apelin receptors. Science 343, 1248636 (2014).

25. Perez-Camps, M. et al. Quantitative imaging reveals real-time Pou5f3-Nanog complexes driving dorsoventral mesendoderm patterning in zebrafish. elife $\mathbf{5}$, e11475 (2016).

26. Deng, C., Chen, H., Yang, N., Feng, Y. \& Hsueh, A. J. Apela regulates fluid homeostasis by binding to the APJ receptor to activate Gi signaling. J. Biol. Chem. 290, 18261-18268 (2015).
27. Chen, $\mathrm{H}$. et al. ELABELA and an ELABELA fragment protect against AKI. J. Am. Soc. Nephrol. 28, 2694-2707 (2017).

28. Schreiber, C. A., Holditch, S. J., Generous, A. \& Ikeda, Y. Sustained ELABELA gene therapy in high-salt diet-induced hypertensive rats. Curr. Gene Ther. 16, 349-360 (2017).

29. Sato, T. et al. ELABELA-APJ axis protects from pressure overload heart failure and angiotensin II-induced cardiac damage. Cardiovasc. Res. 113, 760-769 (2017).

30. Yang, X. \& Gu, D. Response by Yang and Gu to letter regarding article, "predicting the 10-year risks of atherosclerotic cardiovascular disease in Chinese Population: the China-PAR project (prediction for ASCVD risk in China)". Circulation 135, e822-e823 (2017).

31. Zhu, Q. et al. Overexpression of HIF prolyl-hydoxylase-2 transgene in the renal medulla induced a salt sensitive hypertension. J. Cell. Mol. Med. 16, 2701-2707 (2012).

32. Wang, L. et al. Sodium butyrate suppresses angiotensin I-induced hypertension by inhibition of renal (pro)renin receptor and intrarenal renin-angiotensin system. J. Hypertens. 35, 1899-1908 (2017).

33. Zhang, L. et al. Sodium butyrate attenuates angiotensin I-induced cardiac hypertrophy by inhibiting COX2/PGE2 pathway via a HDAC5/HDAC6dependent mechanism. J. Cell. Mol. Med. 23, 8139-8150 (2019).

34. Panday, A., Sahoo, M. K., Osorio, D. \& Batra, S. NADPH oxidases: an overview from structure to innate immunity-associated pathologies. Cell. Mol. Immunol. 12, 5-23 (2015).

35. Veskoukis, A. S., Margaritelis, N. V., Kyparos, A., Paschalis, V. \& Nikolaidis, M. G. Spectrophotometric assays for measuring redox biomarkers in blood and tissues: the NADPH network. Redox Rep. 23, 47-56 (2018).

36. Hashimoto, T. et al. Requirement of apelin-apelin receptor system for oxidative stress-linked atherosclerosis. Am. J. Pathol. 171, 1705-1712 (2007).

37. Zhao, Y. et al. Superoxide anions modulate the performance of apelin in the paraventricular nucleus on sympathetic activity and blood pressure in spontaneously hypertensive rats. Peptides 121, 170051 (2019).

38. Huang, S. K., Shin, K., Sarker, M. \& Rainey, J. K. Apela exhibits isoform- and headgroup-dependent modulation of micelle binding, peptide conformation and dynamics. Biochim. Biophys. Acta Biomembr 1859, 767-778 (2017).

39. Li, P. L. Cardiovascular pathobiology of inflammasomes: inflammatory machinery and beyond. Antioxid. Redox Signal. 22, 1079-1083 (2015).

40. Shahzad, K. et al. Nlrp3-inflammasome activation in non-myeloid-derived cells aggravates diabetic nephropathy. Kidney Int. 87, 74-84 (2015).

41. Chaiworapongsa, T., Chaemsaithong, P., Yeo, L. \& Romero, R. Pre-eclampsia part 1: current understanding of its pathophysiology. Nat. Rev. Nephrol. 10 466-480 (2014)

42. Reuter, S., Gupta, S. C., Chaturvedi, M. M. \& Aggarwal, B. B. Oxidative stress, inflammation, and cancer: how are they linked? Free Radic. Biol. Med. 49, 1603-1616 (2010)

43. Zhou, Q., Cao, J. \& Chen, L. Apelin/APJ system: a novel therapeutic target for oxidative stress-related inflammatory diseases (review). Int. J. Mol. Med. 37, 1159-1169 (2016).

44. Foussal, C. et al. Activation of catalase by apelin prevents oxidative stress-linked cardiac hypertrophy. FEBS Lett. 584, 2363-2370 (2010).

45. Koguchi, W. et al. Cardioprotective effect of apelin-13 on cardiac performance and remodeling in end-stage heart failure. Circ. J. 76, 137-144 (2012).

46. Xie, F. et al. The endoplasmic reticulum stress-autophagy pathway is involved in apelin-13-induced cardiomyocyte hypertrophy in vitro. Acta Pharmacol. Sin. 38, 1589-1600 (2017).

47. Liu, M. et al. ROS-Autophagy pathway mediates monocytes-human umbilical vein endothelial cells adhesion induced by apelin-13. J. Cell. Physiol. 233, 6839-6850 (2018).

48. Ji, M. et al. Targeting hypertension: superoxide anions are involved in apelininduced long-term high blood pressure and sympathetic activity in the paraventricular nucleus. Curr. Neurovasc. Res. 16, 455-464 (2019).

49. Freyer, L. et al. Loss of apela peptide in mice causes low penetrance embryonic lethality and defects in early mesodermal derivatives. Cell Rep. 20 2116-2130 (2017) 